\title{
Nucleation of colloids and macromolecules in a finite volume
}

\author{
James F. Lutsko ${ }^{1}$ \\ Center for Nonlinear Phenomena and Complex Systems, Code Postal 231, \\ Université Libre de Bruxelles, Blvd. du Triomphe, 1050 Brussels, \\ Belgiuma)
}

(Dated: 10 March 2018)

A recently formulated description of homogeneous nucleation for Brownian particles in the over-damped limit based on fluctuating hydrodynamics is used to determine the nucleation pathway, characterized as the most likely path (MLP), for the nucleation of a dense-concentration droplet of globular protein from a dilute solution in a small, finite container. The calculations are performed by directly discretizing the equations for the MLP and it is found that they confirm previous results obtained for infinite systems: the process of homogeneous nucleation begins with a long-wavelength, spatially-extended concentration fluctuation that it condenses to form the pre-critical cluster. This is followed by a classical growth processes. The calculations show that the post-critical growth involves the formation of a depletion zone around the cluster whereas no such depletion is observed in the pre-critical cluster. The approach therefore captures dynamical effects not found in classical Density Functional Theory studies while consistently describing the formation of the pre-critical cluster.

a)Electronic mail: jlutsko@ulb.ac.be; http://www.lutsko.com 


\section{INTRODUCTION}

Recently, the process of diffusion-limited nucleation has been formulated within the context of fluctuating hydrodynamics $\underline{1-3}$. This approach gives a unified description of the formation and growth of an unstable, pre-critical nucleus by means of thermal fluctuations as well as of the post-critical growth driven by a free energy gradient. In this dynamical approach to the description of nucleation, the fundamental quantity is a hydrodynamic field, the local concentration ${ }^{4}$. It can be used to derive the elements of Classical Nucleation Theory $(\mathrm{CNT})^{2,}, \underline{5}$ when the concentration-field is parameterized using the usual capillary approximation that is implicit in CNT. More complex parameterizations are possible giving thereby a more fine-grained description of the nucleation pathway. By this means, it was found that the dynamical theory predicts an unexpected, non-classical pathway wherein nucleation is initiated by a long-wavelength, small-amplitude concentration fluctuation. Spatial localization of the mass in the fluctuation leads to the formation of a relatively large pre-critical cluster. This is followed by a second stage consisting of growth of the pre-critical cluster in accordance with more classical approaches (CNT, see, e.g., Ref. 6, Density functional theory (DFT), see, e.g., Refs.7-10, ...) One advantage of the dynamical approach is that it can be used to determine the relative likelihood of different proposed nucleation pathways and it was found that the non-classical path is much more likely than those of more classical approaches, such as DFT, which inevitably show cluster formation as a spatially-localized processes $^{3}$.

All of the results described above were obtained for an infinite system. This leaves open the question as to whether the non-classical path will dominate in a finite system more typical of a real experiment. Since the physics of the non-classical path is rooted in mass conservation, it seems possible that in a finite system with finite mass, a different result could emerge. This could be particularly relevant in very small systems such as those typical of nano-fluidics, biological processes and lab-on-a-chip applications. A second question left open in previous studies is whether the use of parameterized profiles, no matter how complex, might be prejudicing the results. The purpose of this paper is to address both of these issues. Calculations based on the dynamical description of nucleation are presented for the nucleation of a bubble of dense protein-rich solution from a mother-phase of dilute protein solution in a small, finite volume. The calculations are performed by directly integrating a 
discretized version of the field equations that determine the most likely path in concentrationfield space connecting the initial, nearly uniform dilute phase and a final state consisting of a large, stable droplet of dense solution within a small spherical cavity. It is found that the two-step mechanism is preserved in modified form and that further details of the process that were inaccessible with the parameterized models, such as the formation of a depletion zone during post-critical growth, are evident. Further, the additional resolution afforded by the direct discretization of the equations governing the nucleation pathway, as opposed to the use of parameterizations, allows to further distinguish the results of the dynamical theory from those of more standard DFT-based approaches.

The next section presents the basic theory describing nucleation in terms of a temporally evolving concentration field. The stochastic description is discretized and the determination of the initial state, critical cluster, final state and most likely pathway between them is formulated. The third section presents calculations for the nucleation of protein-rich model globular protein from a dilute mother phase. The paper concludes with a discussion of the results compared to those obtained by other approaches and with some remarks concerning open questions.

\section{THEORY}

\section{A. Stochastic evolution of the concentration}

The fundamental quantity in the present formulation is the local number concentration $\rho(\mathbf{r})$. In the over-damped limit, the evolution of the local concentration is governed by the stochastic differential equation 1,2 (SDE)

$$
\frac{\partial \rho(\mathbf{r} ; t)}{\partial t}=D \boldsymbol{\nabla} \rho(\mathbf{r} ; t) \boldsymbol{\nabla}\left(\frac{\delta F[\rho]}{\delta \rho(\mathbf{r})}\right)_{\rho(\mathbf{r}) \rightarrow \rho(\mathbf{r} ; t)}+\nabla \sqrt{2 D \rho(\mathbf{r} ; t)} \boldsymbol{\xi}(\mathbf{r} ; t)
$$

where the noise, $\boldsymbol{\xi}(\mathbf{r} ; t)$, is delta-correlated in both space and time. Even though this SDE involves a state-dependent noise amplitude, it can be shown ${ }^{2}$ that it is Ito-Stratonovich equivalent so that there is no ambiguity in its interpretation. The functional $F[\rho]$ is a coarse-grained free energy. In general, it is not equivalent to the free energy functional of DFT as the latter is an equilibrium quantity. However, it is generally assumed that the coarse-grained quantity is well-approximated by a mean-field approximation. Just to make 
clear the connection, one expects that averaging Eq.(11) over the noise would give something like the usual DDFT form

$$
\frac{\partial\langle\rho(\mathbf{r} ; t)\rangle}{\partial t}=\widetilde{D} \boldsymbol{\nabla}\langle\rho(\mathbf{r} ; t)\rangle \boldsymbol{\nabla}\left(\frac{\delta \widetilde{F}[\rho]}{\delta \rho(\mathbf{r})}\right)_{\rho(\mathbf{r}) \rightarrow\langle\rho(\mathbf{r} ; t)\rangle}
$$

where, now, $\widetilde{F}[\rho]$ is indeed the equilibrium free energy functional of DFT. For spherically symmetric problems, the SDE can be reduced to

$$
\frac{\partial m(r ; t)}{\partial t}=D 4 \pi r^{2} \rho(r ; t) \frac{\partial}{\partial r}\left(\frac{\delta F[\rho]}{\delta \rho(\mathbf{r})}\right)_{\rho(\mathbf{r}) \rightarrow \rho(r ; t)}+\sqrt{8 \pi r^{2} D \rho(r ; t)} \boldsymbol{\xi}(r ; t)
$$

where the spherically symmetric noise, $\boldsymbol{\xi}(r ; t)$, is delta-correlated in both variables ${ }^{1} \underline{2}$. Here, the quantity $m(r ; t)$ is the total mass within a ball of radius $r$,

$$
m(r ; t)=\int_{0}^{r} 4 \pi r^{\prime 2} \rho\left(r^{\prime} ; t\right) d r^{\prime}
$$

For the free energy functional, a simple squared-gradient model will be used

$$
F[\rho]=\int\left\{f(\rho(\mathbf{r}))+\frac{1}{2} K(\nabla \rho(\mathbf{r}))^{2}\right\} d \mathbf{r}
$$

where $f(\rho)$ is the bulk Helmholtz free energy per unit volume. Finally, it is interesting to note that the equation of motion can be written in the particularly simple and illuminating form

$$
\frac{\partial m(r ; t)}{\partial t}=-\left.D \frac{\partial m(r ; t)}{\partial r} \frac{\delta F[\rho]}{\delta m(r)}\right|_{m(r) \rightarrow m(r ; t)}+\sqrt{2 D \frac{\partial m(r ; t)}{\partial r}} \boldsymbol{\xi}(r ; t)
$$

This shows that, in terms of the mass variable, the dynamics is gradient-driven with a fluctuation-dissipation relation.

\section{B. Discretization}

\section{Variables}

In general, SDE's of the form given here are only well-defined once they are discretized. Furthermore, discretization is necessary for practical calculations. Here, space will be discretized via points $r_{i}=i \Delta$ and the boundary of the finite volume of the container under consideration will be at $r_{N}=(N+1 / 2) \Delta$. The masses at those points are $m\left(r_{i}\right) \equiv m_{i}$ for 
$0 \leq i \leq N+1$ and, of course, $m_{0}=0$. The concentration will be represented at intermediate points as

$$
\rho\left(r_{i}+\frac{1}{2} \Delta\right) \equiv \rho_{i+1 / 2}, 0 \leq i \leq N
$$

so that the relation with the mass is

$$
m_{i}=\Delta \sum_{j=0}^{i-1} 4 \pi r_{j+1 / 2}^{2} \rho_{j+1 / 2} \longleftrightarrow 4 \pi r_{i+1 / 2}^{2} \rho_{i+1 / 2}=\frac{m_{i+1}-m_{i}}{\Delta}
$$

Note that the exact linear relation between the masses and densities means that these variables are completely equivalent. Similarly, one has that

$$
\frac{\partial}{\partial m_{i}}=\frac{1}{\Delta 4 \pi}\left(\frac{1}{r_{i-1 / 2}^{2}} \frac{\partial}{\partial \rho_{i-1 / 2}}-\frac{1}{r_{i+1 / 2}^{2}} \frac{\partial}{\partial \rho_{i+1 / 2}}\right) .
$$

With this discretization, the variable $\rho_{N+1 / 2}$ is the concentration at the wall and $m_{N+1}$ is the total mass within the container. Since mass is conserved, the value of $m_{N+1}$ is, by definition, constant and that of $\rho_{N+1 / 2}$ is determined by mass conservation and the other densities via $\mathrm{Eq} \cdot(8)$.

\section{The free energy}

The free energy is discretized using the same integration scheme as relates the densities and masses,

$$
\beta F=\Delta \sum_{j=0}^{\infty} 4 \pi r_{j+1 / 2}^{2} \beta f\left(\rho_{j+1 / 2}\right)+\Delta \sum_{j=0}^{\infty} 4 \pi \frac{1}{2} K\left(r \frac{\partial \rho}{\partial r}\right)_{j+1 / 2}^{2}
$$

Note that the upper limits on the sums are infinite. This is because a boundary condition on the concentration is needed in order to be able to evaluate the square-gradient term at the boundary of the container. To this end, it will be assumed that outside the container, there is a uniform concentration denoted $\rho_{\infty}$ so that, formally, $\rho_{i+1 / 2}=\rho_{\infty}$ for $i \geq N$. This concentration could be zero, in the case of a truly isolated system, or could be set to a finite value in order, e.g., to approximate nucleation in an infinite system. As it stands, the squared gradient term requires the concentration derivative at intermediate points. This is approximated as

$$
\left(r \frac{\partial \rho}{\partial r}\right)_{j+1 / 2}^{2}=\frac{1}{2}\left(\left(r \frac{\partial \rho}{\partial r}\right)_{j}^{2}+\left(r \frac{\partial \rho}{\partial r}\right)_{j+1}^{2}\right)+O(\Delta)
$$


giving

$$
\begin{aligned}
\beta F & =\Delta \sum_{j=0}^{\infty} 4 \pi r_{j+1 / 2}^{2} \beta f\left(\rho_{j+1 / 2}\right)+\Delta \sum_{j=0}^{\infty} 2 \pi K\left(r \frac{\partial \rho}{\partial r}\right)_{j}^{2} \\
& =\Delta \sum_{j=0}^{\infty} 4 \pi r_{j+1 / 2}^{2} \beta f\left(\rho_{j+1 / 2}\right)+\Delta \sum_{j=0}^{\infty} 2 \pi K r_{j}^{2}\left(\frac{\rho_{j+1 / 2}-\rho_{j-1 / 2}}{\Delta}\right)^{2}
\end{aligned}
$$

or, with the boundary condition,

$$
\beta F-\beta F_{\infty}=\Delta \sum_{j=0}^{N} 4 \pi r_{j+1 / 2}^{2} \beta f\left(\rho_{j+1 / 2}\right)+\Delta \sum_{j=0}^{N+1} 2 \pi K r_{j}^{2}\left(\frac{\rho_{j+1 / 2}-\rho_{j-1 / 2}}{\Delta}\right)^{2}
$$

where $\beta F_{\infty}$ is the energy of the system with uniform concentration $\rho_{\infty}$.

\section{The stochastic differential equation}

With this discretization, the SDE becomes

$$
\frac{\partial m_{i}(t)}{\partial t}=-D \frac{m_{i+1}(t)-m_{i-1}(t)}{2 \Delta} \frac{\partial F}{\partial \Delta m_{i}(t)}+\sqrt{2 D \frac{m_{i+1}(t)-m_{i-1}(t)}{2 \Delta}} \sqrt{\frac{1}{\Delta}} \boldsymbol{\xi}_{i}(t), \quad 1 \leq i \leq N
$$

with $\left\langle\boldsymbol{\xi}_{i}(t) \boldsymbol{\xi}_{j}\left(t^{\prime}\right)\right\rangle=\delta\left(t-t^{\prime}\right) \delta_{i j}$. There are only equations for $m_{i}$ for $i$ up to $N$ since, as

discussed above, the wall is at position $N+1 / 2$ and the total mass in the container, $m_{N+1}$, is conserved. Using Eq.(8) and (9) gives

$$
\frac{d \rho_{i+1 / 2}}{d t}=\frac{1}{4 \pi r_{i+1 / 2}^{2} \Delta}\left(\left(1-\delta_{i N}\right) \frac{d m_{i+1}}{d t}-\left(1-\delta_{i 0}\right) \frac{d m_{i}}{d t}\right), 0 \leq i \leq N .
$$

where it the invariance of $m_{N+1}$ and of $m_{0}=0$ have been explicitly indicated. Expanding, this gives,

$$
\frac{\partial \rho_{i+1 / 2}(t)}{\partial t}=-D \sum_{j=0}^{N} g_{i j}^{-1} \frac{\partial F}{\partial \rho_{j+1 / 2}(t)}+\sqrt{2 D} \sum_{j=0}^{N} q_{i j}^{-1} \xi_{j}(t), \quad 0 \leq i \leq N
$$

with

$$
\begin{aligned}
g_{i j}^{-1} & =\left(1-\delta_{i N}\right) \frac{r_{i+3 / 2}^{2} \rho_{i+3 / 2}+r_{i+1 / 2}^{2} \rho_{i+1 / 2}}{8 \pi r_{i+1 / 2}^{2} \Delta^{3}}\left(\frac{1}{r_{i+1 / 2}^{2}} \delta_{i j}-\frac{1}{r_{i+3 / 2}^{2}} \delta_{i+1 j}\right) \\
& -\left(1-\delta_{i 0}\right) \frac{r_{i+1 / 2}^{2} \rho_{i+1 / 2}+r_{i-1 / 2}^{2} \rho_{i-1 / 2}}{8 \pi r_{i+1 / 2}^{2} \Delta^{3}}\left(\frac{1}{r_{i-1 / 2}^{2}} \delta_{i-1 j}-\frac{1}{r_{i+1 / 2}^{2}} \delta_{i j}\right), 0 \leq i, j \leq N
\end{aligned}
$$


and

$$
q_{i j}^{-1}=\frac{1}{4 \pi \sqrt{2} \Delta^{3 / 2}} \frac{\left(1-\delta_{i N}\right) \delta_{i+1 j}-\left(1-\delta_{i 0}\right) \delta_{i j}}{r_{i+1 / 2}^{2}} \sqrt{r_{j+1 / 2}^{2} \rho_{j+1 / 2}+r_{j-1 / 2}^{2} \rho_{j-1 / 2}}, \quad 0 \leq i, j \leq N
$$

It is easy to check that the fluctuation-dissipation relation $\mathbf{g}^{-1}=\mathbf{q} \mathbf{q}^{T}$ holds and that the discretized equation remains Ito-Stratonovich equivalent 11 .

Just as one of the mass variables is fixed by the constraint of constant mass, so one of the concentration variables is also redundant. In fact, from the previous equation it follows, since the total mass is conserved, that

$$
\frac{d \rho_{N+1 / 2}}{d t}=\frac{1}{4 \pi r_{i+1 / 2}^{2} \Delta}\left(\frac{d m_{N+1}}{d t}-\frac{d m_{N}}{d t}\right)=-\frac{1}{4 \pi r_{i+1 / 2}^{2} \Delta} \frac{d m_{N}}{d t}
$$

There are two possibilities: either one proceeds using this as the equation of motion or one eliminates the variable $\rho_{N+1 / 2}$ altogether by means of the expression for the total mass giving

$$
r_{N+1 / 2}^{2} \rho_{N+1 / 2}=\frac{1}{4 \pi} m_{N+1}-\sum_{i=0}^{N-1} r_{i+1 / 2}^{2} \rho_{i+1 / 2}
$$

so that in fact the remaining variables are the masses for $1 \leq j \leq N$ or, equivalently, the densities up to $\rho_{N-1 / 2}$. When evaluating derivatives, one must then use, e.g.,

$$
\begin{aligned}
\frac{\partial \beta F}{\partial \rho_{i+1 / 2}} & =\left.\frac{\partial \beta F}{\partial \rho_{i+1 / 2}}\right|_{\rho_{N+1 / 2}}+\frac{\partial \rho_{N+1 / 2}}{\partial \rho_{i+1 / 2}} \frac{\partial \beta F}{\partial \rho_{N+1 / 2}} \\
& =\left.\frac{\partial \beta F}{\partial \rho_{i+1 / 2}}\right|_{\rho_{N+1 / 2}}-\frac{r_{i+1 / 2}^{2}}{r_{N+1 / 2}^{2}} \frac{\partial \beta F}{\partial \rho_{N+1 / 2}}
\end{aligned}
$$

The notation indicates that in the first term on the right hand side the derivatives are evaluated holding $\rho_{N+1 / 2}$, as well as all other concentration $\rho_{j+1 / 2}$ except for $j=i$, constant. Note that the second term on the right plays no role when discretizing the SDE, Eq.(16).

\section{Stationary solutions}

A special role will be played by the stationary solutions to the deterministic dynamics (i.e. the dynamics with no noise). These will include both the metastable initial and final states and the metastable critical cluster separating them. They are simply the solutions of

$$
\frac{\partial F}{\partial m_{i}}=0, \quad 1 \leq i \leq N
$$


under the constraint that the total mass is conserved (so that all derivatives are understood to be evaluated with $m_{N+1}$ fixed). Equivalently, in terms of the concentration this gives

$$
\frac{1-\delta_{i N}}{4 \pi r_{i+1 / 2}^{2}} \frac{\partial F}{\partial \rho_{i+1 / 2}}-\frac{1}{4 \pi r_{i-1 / 2}^{2}} \frac{\partial F}{\partial \rho_{i-1 / 2}}=0,1 \leq i \leq N .
$$

where the derivatives are evaluated as indicated in Eq.(21), so that this could also be written as

$$
\begin{aligned}
&\left.\frac{1}{4 \pi r_{i-1 / 2}^{2}} \frac{\partial F}{\partial \rho_{i-1 / 2}}\right|_{\rho_{N+1 / 2}}=\left.\frac{1}{4 \pi r_{i+1 / 2}^{2}} \frac{\partial F}{\partial \rho_{i+1 / 2}}\right|_{\rho_{N+1 / 2}}, 1 \leq i \leq N-1 \\
&\left.\frac{1}{4 \pi r_{N-1 / 2}^{2}} \frac{\partial F}{\partial \rho_{N-1 / 2}}\right|_{\rho_{N+1 / 2}}=\frac{1}{4 \pi r_{N+1 / 2}^{2}} \frac{\partial F}{\partial \rho_{N+1 / 2}} .
\end{aligned}
$$

Finally, by introducing an additional variable, $\mu$, these $N$ equations can be replaced by the $N+1$ equations

$$
\begin{array}{r}
\left.\frac{\partial F}{\partial \rho_{i-1 / 2}}\right|_{\rho_{j+1 / 2}}=4 \pi r_{i-1 / 2}^{2} \mu, 1 \leq i \leq N \\
m_{N+1}=\Delta \sum_{j=0}^{N} 4 \pi r_{j+1 / 2}^{2} \rho_{j+1 / 2}
\end{array}
$$

for the variables $\rho_{i+1 / 2}, 0 \leq i \leq N$ and $\mu$. In this form, it is clear that the stationary solutions simply correspond to extrema of the free energy functional subject to the condition of constant mass, as one would expect. A convenient method of solution of these equations is given in the Appendix A.

\section{Nucleation pathway}

In principle, one could simulate the discretized SDE but the goal here is to more directly characterize the nucleation pathway. Since the problem of interest is the nucleation of a dense phase, with average bulk concentration $\rho_{l}$, from a dilute phase with average bulk concentration $\rho_{v}$, one approach to characterizing the nucleation pathway is to look for the most likely path $\underline{\underline{1}} \underline{\underline{3}}$ connecting an initial state with $\rho(r ; t=0) \sim \rho_{v}$ and final state $\rho(r ; t=T) \sim \rho_{l}$. For an infinite system, these conditions would be strict equivalences but for a finite system, there will in general be spatial variation of the concentration, even in the initial state due to the boundaries. Furthermore, for a finite container, since mass is 
conserved, there cannot be a complete conversion from the initial state $\rho(r ; t=0) \sim \rho_{v}$ to the final concentration, $\rho_{l}$ : the lowest energy state that conserves mass will consist of a droplet inside of which the concentration is roughly that of the final state, $\rho_{l}$, and outside of which the concentration decays to some value that may or may not be near $\rho_{v}$ depending on the boundary conditions.

It has previously been shown that in the weak noise limit, in which the thermodynamic driving force is large compared to typical noise fluctuations, the most likely path connecting

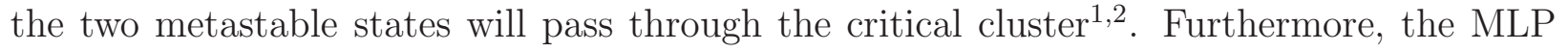
can be determined by following the deterministic equations of motion forward and backward in time. In practice, this means that one starts at the critical cluster and then perturbs the system slightly, first in the direction of one metastable state and then in the direction of the other, and follows the deterministic dynamics given in the present case by

$$
\frac{\partial m_{i}(t)}{\partial t}=-D \frac{m_{i+1}(t)-m_{i-1}(t)}{2 \Delta} \frac{\partial F}{\partial \Delta m_{i}(t)}, \quad 1 \leq i \leq N
$$

or, equivalently,

$$
\frac{\partial \rho_{i+1 / 2}(t)}{\partial t}=-D \sum_{j=0}^{N} g_{i j}^{-1} \frac{\partial F}{\partial \rho_{j+1 / 2}(t)}, \quad 0 \leq i \leq N-1
$$

where, again, the derivatives on the right are evaluated using Eq.(21). There are several important points to be made about this procedure. First, even though there is no noise term in Eq.( 26), this is a noise driven process: Eq.(26) is simply a convenient and exact means of determining the most likely path which is obviously a concept rooted in the fact that the full dynamics includes fluctuations ${ }^{1,2}$. Second, the fact that one follows the path in both directions away from the critical cluster, or equivalently, that one follows the dynamics forward and backwards in time, does not in any way imply time-reversal invariance. In fact, the underlying dynamics is dissipative and so is definitively not time-reversal invariant. Again, the fact that this procedure serves to determine the MLP is a mathematical result that does not imply anything concerning the time-reversal properties of the actual stochastic dynamics. Finally, the first part of the prescription, namely perturbing away from the critical cluster, must be made more precise. One must perturb away from the initial state because the deterministic driving force in the critical cluster is zero: it is an unstable stationary point of the dynamics. Thus, in principle one must move an infinitesimal distance away from it to initiate the dynamics, but in practise one must of course make a finite perturbation with 
the understanding that the final results are approximate and approach the exact result only as the size of the initial perturbation goes to zero. To construct the perturbation, one first solves a generalized eigenvalue problem as described in Appendix B which typically results in the identification of a single, unstable eigenvalue. The associated eigenvector, $v_{i}$, defines the unstable direction and the initial state is simply

$$
\rho_{i+1 / 2}=\rho_{i+1 / 2}^{*} \pm \epsilon v_{i}, \quad 0 \leq i \leq N-1
$$

where $\epsilon$ is a parameter controlling the size of the imposed perturbation.

The next step is the numerical integration of Eq.(27). Because of the Ito-Stratonovich equivalence of the (spatially-)discretized SDE there is no constraint on the discretization scheme used for the time variable. For example, with discrete time variables $t_{i}=i \tau$, the left hand side is simply $\left(\rho_{i+1 / 2}\left(t_{j+1}\right)-\rho_{i+1 / 2}\left(t_{j}\right)\right) / \tau$ while the right hand side can be evaluated at $t_{j}$, giving an Euler scheme, or at $t_{j}+\tau / 2$ giving a more efficient implicit scheme. Further details are given in Appendix $\mathbb{C}$.

Finally, it is convenient to introduce a parameterization for the nucleation pathway. As described elsewhere ${ }^{2}, \underline{3}$, the structure of the stochastic model induces a natural measure of distance in concentration space between the concentration at time $t_{1}$ and that at time $t_{2}$ given by

$$
d\left[\rho_{1}, \rho_{2}\right]=\int_{t_{1}}^{t_{2}} \sqrt{\int_{0}^{\infty} \frac{1}{4 \pi r^{2} \rho(r ; t)}\left(\frac{\partial m(r ; t)}{\partial r}\right)^{2} d r} d t
$$

By definition, this distance increases monotonically along any proposed nucleation pathway and therefore serves as a natural, induced reaction coordinate.

\section{EXAMPLE: NUCLEATION OF DENSE PROTEIN SOLUTION FROM DILUTE SOLUTION}

In order to illustrate the theory developed above, detailed calculations for a model globular protein ${ }^{12}$ have been performed. The effect of the solvent was approximated, crudely, by assuming Brownian dynamics of the (large) solute molecules which also experience an effective pair interaction for which the ten Wolde-Frenkel interaction potential 13

$$
v(r)=\left\{\begin{array}{c}
\infty, \quad r \leq \sigma \\
\frac{4 \epsilon}{\alpha^{2}}\left(\left(\frac{1}{\left(\frac{r}{\sigma}\right)^{2}-1}\right)^{6}-\alpha\left(\frac{1}{\left(\frac{r}{\sigma}\right)^{2}-1}\right)^{3}\right), r \geq \sigma
\end{array}\right.
$$




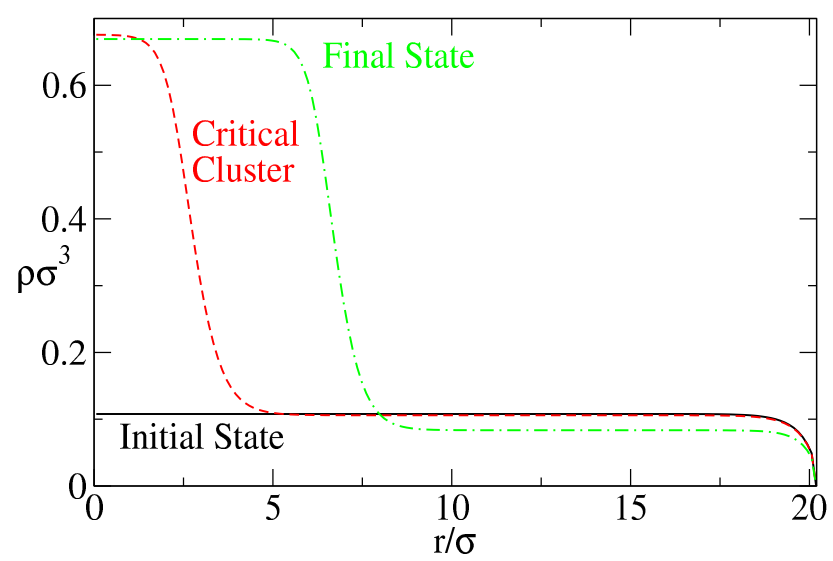

FIG. 1. The concentration distribution in the metastable, nearly uniform initial state (solid line), the critical cluster (broken line) and the final stable cluster (dash-dot line) as a function of distance from the center of the cluster.

was used with $\alpha=50$ which is then cutoff at $r_{c}=2.5 \sigma$ and shifted so that $v\left(r_{c}\right)=0$. The temperature is fixed at $k_{B} T=0.375 \epsilon$ and the equation of state computed using thermodynamic perturbation theory. The transition studied was that between the dilute phase and the dense protein phase which, in the present simplified picture, is completely analogous to the vapor-liquid transition for particles interacting under the given pair potential. The gradient coefficient, $K$, is calculated from the pair potential using the approximation given in Ref. 14

$$
\beta K \simeq-\frac{2 \pi}{45} d^{5} \beta v(d)+\frac{2 \pi}{15} \int_{d}^{\infty}\left(2 d^{2}-5 r^{2}\right) \beta v(r) r^{2} d r
$$

where $d$ is the effective hard-sphere diameter for which the Barker-Henderson approximation was used $\frac{15}{}$. For the temperature used here it was found that $\beta K=1.80322 \sigma^{5}$.

The nucleation pathway has been evaluated for a spherical box with a radius or $20.63 \sigma$ using a lattice of 160 points with a spacing equal to $0.126 \sigma$ and with the external concentration set to zero. The time step used was $10^{-4} D^{-1} \sigma^{2}$. The only remaining control parameter is the initial mass of material in the container. For the chosen temperature, the coexistence densities of the dilute and dense phases are $\rho \sigma^{3}=0.071$ and $\rho \sigma^{3}=0.663$, respectively. The initial condition for the calculations presented here was a uniform concentration which was 
1.5 times the dilute phase coexistence concentration which serves to fix the total mass. The various metastable states were then determined under the constraint that the total mass does not vary. Figure 1 shows the initial metastable state, the critical cluster and the final, stable state. Since the material in the center of the cluster that eventually forms is near the bulk state, it is clear that the if the initial state contains too little matter, no cluster will form. In fact, if the amount of matter in the system is scaled with the radius, then as the radius of the system is reduced, the final stable cluster has smaller and smaller radius until at some point it is smaller than the critical cluster: i.e., there is no barrier separating the initial and final states.

One quantity of particular interest is the size of the cluster. In previous work on infinite systems, this was characterized by both the equimolar radius and by identifying the point at which the difference of the concentration from the background was sufficiently small. In the present case of relatively small, finite systems that conserve mass, the equimolar radius is not very useful so only the second measure is employed. Even then, the "background" concentration has to be reinterpreted: here, it is taken to be the concentration a distance of $2 \sigma$ from the wall (so as to avoid boundary effects), a point denoted as $r_{b}$ since it defines the "background". The radius is then determined by starting at the center of the cluster, i.e. at $r=0$, and increasing $r$ until a point $R$ is found at which

$$
\rho(R)=\rho\left(r_{b}\right)+\epsilon\left(\rho(0)-\rho\left(r_{b}\right)\right)
$$

for some prescribed value of $\epsilon$, typically either $\epsilon=\frac{1}{2}$ (which will be referred to as the half width) or $\epsilon=0.1$ (which will be referred to as the total width).

The determination of the two branches of the pathway was initiated by perturbing in the direction of the unstable eigenvector with the proportionality constant being adjusted so as to produce a change in free energy of $\Delta F=0.005 k_{B} T$ from the critical cluster. Figure 2 shows the evolution of the cluster radius, the central concentration and the free energy difference as functions of distance as well as of the cluster size along the nucleation pathway. The results are similar to those obtained previously for an infinite system using parameterized concentration profiles. In particular, the fact that the process of nucleation involves two separate steps: first, a long wavelength, small amplitude concentration fluctuation forms. This stage is characterized by a large initial radius that decreases while the central concentration increases. At a certain moment, when the concentration is near its bulk value, the 

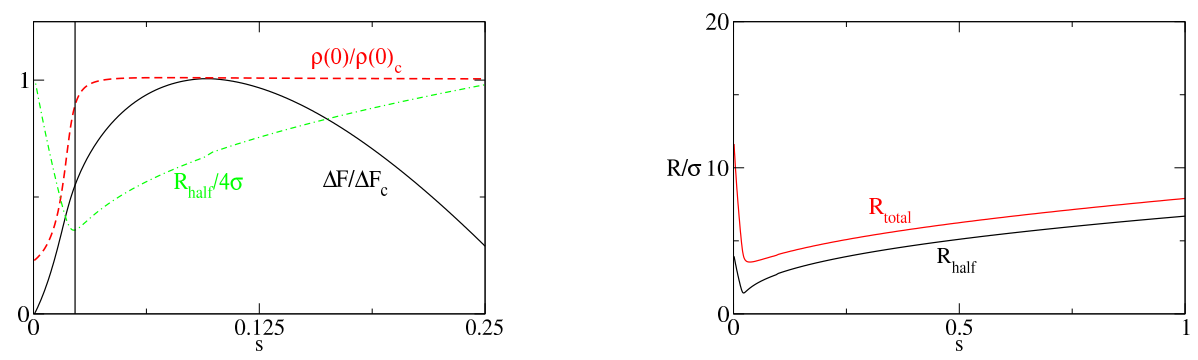

FIG. 2. The left panel shows the free energy difference from the initial state, $\Delta F / \Delta F_{c}$, the concentration at the center of the cluster, $\rho(0) / \rho_{c}(0)$, and the cluster radius, $R_{\text {half }} /(4 \sigma)$, as functions of distance along the nucleation pathway. The energy and concentration are scaled by their values in the critical cluster whereas the radius has been arbitrarily scaled to $4 \sigma$. The vertical line shows the point at which the radius reaches its minimum. The right panel shows two measures of the cluster size as a function of distance along the pathway.

radius begins to increase and, in this second stage, the evolution of the cluster follows the classical scenario of monotonic growth of radius and mass at constant, near-bulk interior concentration.

There is one significant difference in the pre- and post-critical cluster growth as shown in Fig. 3. Growth of the post-critical cluster is deterministic and driven by the fact that the free energy of the system decreases with increasing cluster size so that material is actively drawn into the cluster. The fact that the low-concentration protein drawn into the cluster can only be replaced by the diffusion of new material from further away results in the formation of a region with a lower concentration of protein than in the mother phase, further from the cluster; in other words, in the formation of a depletion zone. The formation of a depletion zone is expected in classical treatments of cluster growth $\underline{16}$. The process is governed by the fact that the lower the concentration in the mother phase, the larger the corresponding critical cluster. If material is drawn into the growing cluster faster than new material can replace it by diffusion, (a situation that is always assumed to occur for sufficiently large clusters since the thermodynamic driving force increases with cluster size), then the cluster will lower the concentration in the adjacent region to the point that the thermodynamic driving force is sufficiently small that the rate of addition of material to the cluster will be 

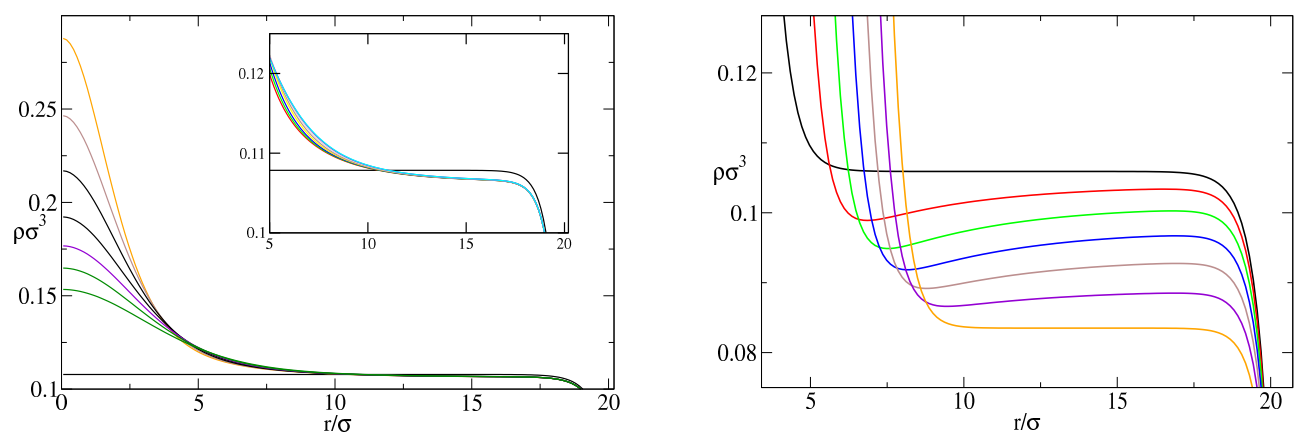

FIG. 3. The evolution of the concentration along the nucleation pathway. The left panel shows the early stages of formation of the pre-critical cluster (increasing concentration at $r=0$ corresponds to increasing distance along the nucleation pathway). The large spatial extent of the initial concentration perturbation is evident and the inset shows the monotonic variation of the concentration as a function of distance from the center. The right panel shows part of the concentration distribution during post-critical growth (the maximum concentration moves to increasing radii as one progresses along the nucleation pathway). The critical cluster, left-most curve, and final state, right-most curve, show no depletion zone, but the presence of a depletion zone in intermediate clusters is clear.

equal to the rate of replenishment by diffusion. Assuming diffusion is slow, this will force the cluster to be very near the critical cluster for the adjacent depleted mother phase.

In contrast to the formation of the depletion zone for post-critical clusters, Fig. 3 shows that no depletion zone exists for pre-critical clusters. The reasoning is the opposite to that for post-critical growth: the pre-critical cluster is unstable and its growth is governed by unlikely fluctuations. If the concentration outside the cluster is unnecessarily low, then the cluster will be even more unstable (since, as pointed out above, lower concentration in the mother phase increases the size of the critical cluster). Hence, the most favorable way to form an unstable cluster is one that does not create any region of lowered concentration. Of course, since mass is conserved, the concentration outside the cluster must decrease as the cluster grows, but, as can be seen in the figure, the concentration is at a maximum just "outside" the cluster (however that is defined) and decreases monotonically as one moves further away from it so that the concentration of the mother phase just outside the cluster is as large as possible, i.e. the maximum of the concentration anywhere in the mother phase. 
This illustrates a point made previously ${ }^{2}$, namely that the initial formation of a cluster as a localized object with small radius would necessarily involve the formation of a depletion zone, due to mass conservation, and this would clearly be unfavorable since it would make any (unstable) pre-critical cluster even more unstable. Instead, what the present results show is that the most likely nucleation pathway involves a long-wavelength concentration fluctuation that contains and localizes enough mass to form the critical cluster.

\section{CONCLUSIONS}

A discretized version of the stochastic field equations for fluctuations in a fluid of Brownian particles in the over-damped limit have been given. The most likely path for nucleation was computed in the weak-noise limit and it was found that the pathway obeyed the same two-stage mechanism as previously found for infinite systems. Namely, nucleation begins with a long-wavelength, small-amplitude fluctuation that evolves by becoming spatially more localized until the concentration inside the fluctuation reaches near-bulk values. This is followed by a second stage of spatial growth of the cluster that is similar to that assumed in CNT.

It was observed that an important difference between pre-critical and post-critical growth is that in the former case, no depletion zone forms whereas in the latter, a depletion zone is clearly in evidence. The existence of depletion zone is not surprising and, indeed, is assumed in classical treatments of cluster growth. However, its presence is important in distinguishing the results of the present, dynamical approach to nucleation from those of other approaches. One alternative method is the use of equilibrium DFT. The determination of the properties of the critical cluster via DFT is relatively uncontroversial, however the use of DFT to determine the pathway, while widely practiced, is harder to justify. This is because DFT is a strictly equilibrium theory $\underline{\underline{10}}$ whereas nucleation is, by its nature, a nonequilibrium, fluctuation-driven processes ${ }^{2}$. It is therefore unclear why DFT should be the right tool to describe the nucleation pathway. This is reflected in the fact that it is difficult to map out the pathway using DFT which is based on minimizing a free energy functional. The problem is that non-critical clusters are obviously not stationary points of the free energy so that one can only determine their properties using DFT if some sort of external constraint is applied $\frac{17-19}{19}$ so as to stabilize them. This leads to the problem that 
there is no unique constraint and different constraints can lead to different results $\underline{14}, 20$.

In answer to this problem, another approach has gained popularity in recent years. It involves the determination of the minimum free energy pathway connecting the critical cluster to the initial and final states ${ }^{20}-25$. While this does not involve the imposition of any constraints, it nevertheless suffers from a similar arbitrariness as the MFEP can only be calculated once a method for determining distances in concentration-space has been $\operatorname{adopted}^{21}$. Again, without further input the choice of metric in concentration space is arbitrary and the method ends up being no better founded than the older constraint-based methods.

Aside from these criticisms of the internal logic of the DFT methods, the present results show a further qualitative failing that demonstrates that free energy considerations, without dynamics, cannot be enough. As discussed above, the formation of a depletion zone is an inevitably consequence of the fact that the thermodynamic driving force for the incorporation of new material into the cluster grows as the cluster grows and must, at some point, cause material to be drawn into the cluster faster than it can be diffusively replaced. None of the DFT studies report seeing a depletion zone for super critical clusters. In all cases, the concentration seems to decay monotonically to the surrounding bulk. The is a clear sign that approaches that do not include dynamics are not sufficient.

An alternative to DFT is its dynamical cousin, Dynamical DFT (DDFT) ${ }^{7}, \underline{10}$. The equations for DDFT are in fact the same as those given above, Eq.(1), if the noise term is dropped or Eq.(3) if it is understood that only forward evolution in time is relevant (for DDFT). The latter point is important: DDFT can only describe a system falling down a free energy surface: by its nature, since it involves no fluctuations, DDFT cannot describe barrier crossing. To overcome this, some studies ${ }^{26}$ have relied on the inclusion of fluctuations in the initial condition for a DDFT calculation but at best this describes a physical system perturbed by an initial, external shock. The results will necessarily be dependent on, e.g., the amplitude and spectrum of the noise and thus is subject to a similar arbitrarity to that of static DFT and for the same reason: both theories are based on equilibrium physics and so cannot naturally describe nonequilibrium states. On the other hand, DDFT does have the virtue that the post-critical growth is correctly described and it will certainly correctly describe the depletion zone.

The dynamical theory presented here and elsewhere does, in some sense, provide an ex- 
tension of DDFT to barrier crossing problems since the MLP is described by DDFT-like equations. However, there are still conceptual differences that separate the two approaches. The most important is the nature of the "free energy" that enters into the dynamical equations. In DDFT, the free energy typically enters via a local equilibrium approximation. Because of the context (i.e. that one is dealing with ensemble-averaged quantities) this is the true, macroscopic free energy functional. In the dynamical approach, the status of the "free energy" is more problematic. It is certainly not the macroscopic free energy. In fact, let us write the DDFT equations in the form

$$
\frac{\partial\langle\rho(\mathbf{r} ; t)\rangle}{\partial t}=D \nabla\langle\rho(\mathbf{r} ; t)\rangle \boldsymbol{\nabla}\left(\frac{\delta \widetilde{F}[\rho]}{\delta \rho(\mathbf{r})}\right)_{\rho(\mathbf{r}) \rightarrow\langle\rho(\mathbf{r} ; t)\rangle}
$$

where the brackets $\langle\ldots\rangle$ indicate an ensemble average and where $\widetilde{F}[\rho]$ is the equilibrium DFT functional. On the other hand, the noise-averaged stochastic equations, Eq.(1), are

$$
\frac{\partial\langle\langle\rho(\mathbf{r} ; t)\rangle\rangle}{\partial t}=D \nabla\left\langle\left\langle\rho(\mathbf{r} ; t) \nabla\left(\frac{\delta F[\rho]}{\delta \rho(\mathbf{r})}\right)_{\rho(\mathbf{r}) \rightarrow \rho(\mathbf{r} ; t)}\right\rangle\right\rangle
$$

where the double brackets $\langle\langle\ldots\rangle\rangle$ indicate an average over the noise. Technically, one cannot identify the ensemble-averaged concentration, $\langle\rho(\mathbf{r} ; t)\rangle$, with the noise-averaged concentration $\langle\langle\rho(\mathbf{r} ; t)\rangle\rangle$ because the noise-averaged concentration also involves a coarse-graining. However, if the spatial variations are sufficiently weak, then it is reasonable to expect these quantities will be more or less the same and so one expects the right hand sides of these equations to become the same. It is then seen that equality of the right hand sides depends on a series of factorization approximations,

$$
\begin{aligned}
\left\langle\left\langle\rho(\mathbf{r} ; t) \nabla\left(\frac{\delta F[\rho]}{\delta \rho(\mathbf{r})}\right)_{\rho(\mathbf{r}) \rightarrow \rho(\mathbf{r} ; t)}\right\rangle\right\rangle & \sim\langle\langle\rho(\mathbf{r} ; t)\rangle\rangle\left\langle\left\langle\nabla\left(\frac{\delta F[\rho]}{\delta \rho(\mathbf{r})}\right)_{\rho(\mathbf{r}) \rightarrow \rho(\mathbf{r} ; t)}\right\rangle\right\rangle \\
& \sim\langle\langle\rho(\mathbf{r} ; t)\rangle\rangle \nabla\left(\frac{\delta \widetilde{F}[\rho]}{\delta \rho(\mathbf{r})}\right)_{\rho(\mathbf{r}) \rightarrow\langle\langle\rho(\mathbf{r} ; t)\rangle\rangle}
\end{aligned}
$$

Interestingly, the assumption of weak noise, which underlies the development of the dynamical approach, might be expected to justify this series of approximations so that a certain consistency between the dynamical theory and DDFT might be seen to emerge. In any case, it is worth noting that in the theory of critical phenomena, it is generally accepted that a mean field approximation of the form hard-core plus mean-field treatment of the attractive part of the potential is a reasonable approximation for the coarse-grained free energy 27 . 
Since this is at present the dominant model used in DFT calculations ${ }^{10}$, it might be argued that the use of this model for the coarse-grained free energy is actually more justifiable than is its use in DFT calculations.

\section{ACKNOWLEDGMENTS}

This work was partially supported in part by the European Space Agency under contract number ESA AO-2004-070 and by FNRS Belgium under contract C-Net NR/FVH 972.

\section{Appendix A: Solution for the critical cluster}

With the discretized free energy, Eq.(13), the equations for the critical cluster, Eq.(25), become

$$
\begin{aligned}
r_{1 / 2}^{2} \beta f^{\prime}\left(\rho_{1 / 2}\right)+K r_{1}^{2}\left(\frac{\rho_{3 / 2}-\rho_{1 / 2}}{\Delta}\right) & =r_{1 / 2}^{2} \mu \quad \text { (A1) } \\
r_{i+1 / 2}^{2} \beta f^{\prime}\left(\rho_{i+1 / 2}\right)+K r_{i}^{2}\left(\frac{\rho_{i+1 / 2}-\rho_{i-1 / 2}}{\Delta}\right)-K r_{i+1}^{2}\left(\frac{\rho_{i+3 / 2}-\rho_{i+1 / 2}}{\Delta}\right) & =r_{1 / 2}^{2} \mu, 1 \leq i \leq N-1 \\
r_{N+1 / 2}^{2} \beta f^{\prime}\left(\rho_{N+1 / 2}\right)+K r_{N}^{2}\left(\frac{\rho_{N+1 / 2}-\rho_{N-1 / 2}}{\Delta}\right)-K r_{N+1}^{2}\left(\frac{\rho_{\infty}-\rho_{N+1 / 2}}{\Delta}\right) & =r_{N+1 / 2}^{2} \mu
\end{aligned}
$$

and

$$
m_{N+1}=4 \pi \sum_{i=0}^{N} r_{i+1 / 2}^{2} \rho_{i+1 / 2} .
$$

To solve these, one begins with a guess, say $\rho_{i+1 / 2}$, and denote the actual solution by $\rho_{i+1 / 2}^{*}$. Defining

$$
\begin{aligned}
x_{i} & =\rho_{i+1 / 2}^{*}-\rho_{i+1 / 2}, 0 \leq i \leq N \\
x_{N+1} & =\mu^{*}-\mu
\end{aligned}
$$

and

$$
\begin{aligned}
y_{i} & =\beta f^{\prime}\left(\rho_{i+1 / 2}\right)+\frac{K}{r_{i+1 / 2}^{2} \Delta}\left(r_{i}^{2}\left(\rho_{i+1 / 2}-\rho_{i-1 / 2}\right)-r_{i+1}^{2}\left(\rho_{i+3 / 2}-\rho_{i+1 / 2}\right)\right), 0 \leq i \leq N-1 \\
y_{N} & =\beta f^{\prime}\left(\rho_{N+1 / 2}\right)+\frac{K}{r_{N+1 / 2}^{2}}\left(r_{N}^{2}\left(\rho_{N+1 / 2}-\rho_{N-1 / 2}\right)-r_{N+1}^{2}\left(\rho_{\infty}-\rho_{N+1 / 2}\right)\right) \\
y_{N+1} & =4 \pi \sum_{i=0}^{N} r_{i+1 / 2}^{2} \rho_{i+1 / 2}-m_{N+1}
\end{aligned}
$$


and expanding to first order gives

$$
\begin{aligned}
-\frac{K}{\Delta}\left(\frac{r_{i}}{r_{i+1 / 2}}\right)^{2} x_{i-1}+ & \left(\beta f^{\prime \prime}\left(\rho_{i+1 / 2}\right)+\frac{K}{r_{i+1 / 2}^{2} \Delta}\left(r_{i}^{2}+r_{i+1}^{2}\right)\right) x_{i} \\
& -\left(1-\delta_{i N}\right) \frac{K}{\Delta}\left(\frac{r_{i+1}}{r_{i+1 / 2}}\right)^{2} x_{i+1}-y_{N+1}=\mu-y_{i}, 0 \leq i \leq N
\end{aligned}
$$

and

$$
4 \pi \sum_{i=0}^{N} r_{i+1 / 2}^{2} x_{i+1 / 2}=y_{N+1} .
$$

These equations are tridiagonal except for the last row and column and can be efficiently solved by a trivial modification of the Thomas algorithm (see, e.g., Ref. ${ }^{28}$ ). Thus, the solution to these equations is found by starting with the boundary conditions, $\rho_{\infty}$ and $m_{N+1}$, and an initial guess, $\rho_{i+1 / 2}$ and $\mu$ (for the latter, a reasonable guess is $f^{\prime}\left(\rho_{\infty}\right)$ ) and then iterating this linear system to stability. Alternatively, one can simply choose a value for $\mu$, eliminate $y_{N+1}$ as a variable (i.e. set $y_{N+1}=0$ in Eq.(A5) ) and drop Eq.(A6) and solve for the values of $x_{i}$. The value of the mass is then evaluated post hoc.

\section{Appendix B: The unstable direction}

To determine the unstable direction, we first note that the deterministic dynamics given in Eq.(27) can be interpreted as being gradient-driven motion in a non-Euclidean geometry $20,21,29$. As such, the unstable direction is determined from the generalized eigenvalue problem

$$
\sum_{j=0}^{N-1} \frac{\partial^{2} F}{\partial \rho_{i+1 / 2} \partial \rho_{j+1 / 2}}=\lambda \sum_{j=0}^{N-1} g_{i j} v_{j}, \quad 0 \leq i \leq N-1
$$

and for convenience we write

$$
g_{i j}^{-1}=-\frac{1}{8 \pi \Delta^{3}} R_{i i^{\prime}}^{-1} \bar{g}_{i^{\prime} j^{\prime}}^{-1} R_{j^{\prime} j}^{-1}
$$

with

$$
R_{i j} \equiv \delta_{i j} r_{i+1 / 2}^{2}
$$

and

$$
\begin{aligned}
\bar{g}_{i j}^{-1} & =\left(1-\delta_{i N}\right)\left(r_{i+3 / 2}^{2} \rho_{i+3 / 2}+r_{i+1 / 2}^{2} \rho_{i+1 / 2}\right)\left(\delta_{i j}-\delta_{i+1 j}\right) \\
& -\left(1-\delta_{i 0}\right)\left(r_{i+1 / 2}^{2} \rho_{i+1 / 2}+r_{i-1 / 2}^{2} \rho_{i-1 / 2}\right)\left(\delta_{i-1 j}-\delta_{i j}\right), \quad 0 \leq i, j \leq N-1
\end{aligned}
$$


which we also write as

$$
\bar{g}_{i j}^{-1}=-\left(a_{i} \delta_{j i-1}+a_{j} \delta_{j i+1}\right)+\left(\left(1-\delta_{i 0}\right) a_{i}+a_{i+1}\right) \delta_{i j}, \quad 0 \leq i, j \leq N-1
$$

where

$$
a_{i}=r_{i+1 / 2}^{2} \rho_{i+1 / 2}+r_{i-1 / 2}^{2} \rho_{i-1 / 2}, \quad 0 \leq i, j<N-1
$$

The inverse of $\mathbf{g}^{-1}$ is easily found to be

$$
\bar{g}_{i j}=\sum_{l=\max (i, j)}^{N-1} \frac{1}{a_{l+1}}
$$

Thus completing specification of the generalized eigenvalue problem.

\section{Appendix C: Integration scheme}

The deterministic equations can be written as

$$
\frac{d \rho_{i+1 / 2}}{d t}=y_{i+1 / 2}\left(\rho_{i-3 / 2}, \rho_{i-1 / 2}, \rho_{i+1 / 2}, \rho_{i+3 / 2}, \rho_{i+5 / 2}\right)
$$

with

$$
\begin{aligned}
& y_{i+1 / 2}\left(\rho_{i-3 / 2}, \rho_{i-1 / 2}, \rho_{i+1 / 2}, \rho_{i+3 / 2}, \rho_{i+5 / 2}\right) \\
& =-\left(1-\delta_{N}\right) \frac{r_{i+3 / 2}^{2} \rho_{i+3 / 2}+r_{i+1 / 2}^{2} \rho_{i+1 / 2}}{8 \pi r_{i+1 / 2}^{2} \Delta^{3}}\left(\frac{1}{r_{i+1 / 2}^{2}} \frac{\partial \beta F}{\partial \rho_{i+1 / 2}}-\frac{1}{r_{i+3 / 2}^{2}} \frac{\partial \beta F}{\partial \rho_{i+3 / 2}}\right) \\
& +\left(1-\delta_{i 0}\right) \frac{r_{i+1 / 2}^{2} \rho_{i+1 / 2}+r_{i-1 / 2}^{2} \rho_{i-1 / 2}}{8 \pi r_{i+1 / 2}^{2} \Delta^{3}}\left(\frac{1}{r_{i-1 / 2}^{2}} \frac{\partial \beta F}{\partial \rho_{i-1 / 2}}-\frac{1}{r_{i+1 / 2}^{2}} \frac{\partial \beta F}{\partial \rho_{i+1 / 2}}\right)
\end{aligned}
$$

and

$\frac{1}{r_{i+1 / 2}^{2}} \frac{\partial \beta F}{\partial \rho_{i+1 / 2}}=\Delta 4 \pi\left(\beta f^{\prime}\left(\rho_{i+1 / 2}\right)+\frac{K}{r_{i+1 / 2}^{2} \Delta^{2}}\left[-r_{i}^{2} \rho_{i-1 / 2}+\left(r_{i}^{2}+r_{i+1}^{2}\right) \rho_{i+1 / 2}-r_{i+1}^{2} \rho_{i+3 / 2}\right]\right)$

Discretizing in time with time step $\delta_{t}$ gives a simple Euler scheme,

$$
\rho_{i+1 / 2}^{(n+1)}-\rho_{i+1 / 2}^{(n)}=\delta_{t} y_{i+1 / 2}^{(n)}
$$

with

$$
y_{i+1 / 2}^{(n)}=y_{i+1 / 2}\left(\rho_{i-3 / 2}^{(n)}, \rho_{i-1 / 2}^{(n)}, \rho_{i+1 / 2}^{(n)}, \rho_{i+3 / 2}^{(n)}, \rho_{i+5 / 2}^{(n)}\right)
$$


Unfortunately, this simple scheme requires a very small time step and is therefore inefficient. As is often the case ${ }^{28}$, the integration can be made much more efficient by introducing an implicit scheme. Define

$$
x_{i+1 / 2}^{(n+1)}=\rho_{i+1 / 2}^{(n+1)}-\rho_{i+1 / 2}^{(n)}
$$

and evaluate the source term at the temporal "midpoint" using

$$
\rho_{i+1 / 2}^{(n+1 / 2)} \equiv \frac{1}{2}\left(\rho_{i+1 / 2}^{(n+1)}+\rho_{i+1 / 2}^{(n)}\right)=\rho_{i+1 / 2}^{(n+1)}-\frac{1}{2} x_{i+1 / 2}^{(n+1)}
$$

and expanding to first order in $x^{(n+1)}$ to get

$$
y_{i+1 / 2}^{(n+1 / 2)} \simeq y_{i+1 / 2}^{(n)}+\sum_{j=-2}^{2} T_{i j}^{(n)} x_{j+1 / 2}^{(n+1)}
$$

with $T_{i j}=T_{j i}$ and

$$
\begin{aligned}
T_{i i}^{(n)} & =-\left(1-\delta_{N}\right) \frac{1}{8 \pi \Delta^{3}}\left(\frac{1}{r_{i+1 / 2}^{2}} \frac{\partial \beta F}{\partial \rho_{i+1 / 2}}-\frac{1}{r_{i+3 / 2}^{2}} \frac{\partial \beta F}{\partial \rho_{i+3 / 2}}\right) \\
& +\left(1-\delta_{i 0}\right) \frac{1}{8 \pi \Delta^{3}}\left(\frac{1}{r_{i-1 / 2}^{2}} \frac{\partial \beta F}{\partial \rho_{i-1 / 2}}-\frac{1}{r_{i+1 / 2}^{2}} \frac{\partial \beta F}{\partial \rho_{i+1 / 2}}\right) \\
& -\left(1-\delta_{N}\right) \frac{r_{i+3 / 2}^{2} \rho_{i+3 / 2}^{(n)}+r_{i+1 / 2}^{2} \rho_{i+1 / 2}^{(n)}}{2 r_{i+1 / 2}^{2}}\left(\frac{1}{\Delta^{2}} \beta f^{\prime \prime}\left(\rho_{i+1 / 2}^{(n)}\right)+\frac{K}{\Delta^{4}}\left(\frac{r_{i}^{2}+r_{i+1}^{2}}{r_{i+1 / 2}^{2}}+\frac{r_{i+1}^{2}}{r_{i+3 / 2}^{2}}\right)\right) \\
& -\left(1-\delta_{i 0}\right) \frac{r_{i+1 / 2}^{2} \rho_{i+1 / 2}^{(n)}+r_{i-1 / 2}^{2} \rho_{i-1 / 2}^{(n)}}{2 r_{i+1 / 2}^{2}}\left(\frac{1}{\Delta^{2}} \beta f^{\prime \prime}\left(\rho_{i+1 / 2}^{(n)}\right)+\frac{K}{\Delta^{4}}\left(\frac{r_{i}^{2}+r_{i+1}^{2}}{r_{i+1 / 2}^{2}}+\frac{r_{i}^{2}}{r_{i-1 / 2}^{2}}\right)\right)
\end{aligned}
$$

and

$$
\begin{aligned}
T_{i i+1}^{(n)} & =-\frac{\left(1-\delta_{N}\right) r_{i+3 / 2}^{2}}{8 \pi r_{i+1 / 2}^{2} \Delta^{3}}\left(\frac{1}{r_{i+1 / 2}^{2}} \frac{\partial \beta F^{(n)}}{\partial \rho_{i+1 / 2}}-\frac{1}{r_{i+3 / 2}^{2}} \frac{\partial \beta F^{(n)}}{\partial \rho_{i+3 / 2}}\right) \\
& +\left(1-\delta_{N}\right) \frac{r_{i+3 / 2}^{2} \rho_{i+3 / 2}^{(n)}+r_{i+1 / 2}^{2} \rho_{i+1 / 2}^{(n)}}{2 r_{i+1 / 2}^{2}}\left(\frac{1}{\Delta^{2}} \beta f^{\prime \prime}\left(\rho_{i+3 / 2}^{(n)}\right)+\frac{K}{\Delta^{4}}\left(\frac{r_{i+1}^{2}}{r_{i+1 / 2}^{2}}+\frac{r_{i+1}^{2}+r_{i+2}^{2}}{r_{i+3 / 2}^{2}}\right)\right) \\
& +\left(1-\delta_{i 0}\right) \frac{r_{i+1 / 2}^{2} \rho_{i+1 / 2}^{(n)}+r_{i-1 / 2}^{2} \rho_{i-1 / 2}^{(n)}}{2 r_{i+1 / 2}^{2}} \frac{K}{\Delta^{4}}\left(\frac{r_{i+1}^{2}}{r_{i+1 / 2}^{2}}\right)
\end{aligned}
$$

and

$$
T_{i i+2}^{(n)}=-\left(1-\delta_{N}\right) \frac{r_{i+3 / 2}^{2} \rho_{i+3 / 2}^{(n)}+r_{i+1 / 2}^{2} \rho_{i+1 / 2}^{(n)}}{r_{i+1 / 2}^{2}} \frac{K}{2 \Delta^{4}}\left(\frac{r_{i+2}^{2}}{r_{i+3 / 2}^{2}}\right) .
$$

The integration scheme then becomes

$$
x_{i+1 / 2}^{(n+1)}=\delta_{t} y_{i+1 / 2}^{(n)}+\delta_{t} \sum_{j=-2}^{2} T_{i j}^{(n)} x_{j+1 / 2}^{(n+1)}
$$


Because of the banded nature of these equations, they can be solved quite efficiently and the resulting integration scheme is much more stable than the simple Euler scheme.

\section{REFERENCES}

${ }^{1}$ J. F. Lutsko, J. Chem. Phys. 135, 161101 (2011).

${ }^{2}$ J. F. Lutsko, J. Chem. Phys. 136, 034509 (2012).

${ }^{3}$ J. F. Lutsko, J. Chem. Phys. 136, 134502 (2012).

${ }^{4}$ The term "concentration" is used throughout this paper since the theory applies to particles in solution that can be modeled by Brownian dynamics. However, since the solvent is not explicitly modeled, there is in fact only one species present so that one could equally well use the term "density".

${ }^{5}$ J. F. Lutsko and M. A. Durán-Olivencia, "Classical nucleation theory from a dynamical approach to nucleation," (2012).

${ }^{6}$ D. Kashchiev, Nucleation : basic theory with applications (Butterworth-Heinemann, Oxford, 2000).

${ }^{7}$ R. Evans, Adv. Phys. 28, 143 (1979).

${ }^{8}$ D. W. Oxtoby and R. Evans, J. Chem. Phys. 89, 7521 (1988).

${ }^{9}$ V. Talanquer and D. W. Oxtoby, J. Chem. Phys. 100, 5190 (1994).

${ }^{10}$ J. F. Lutsko, Adv. Chem. Phys. 144, 1 (2010).

${ }^{11}$ C. W. Gardiner, Handbook of Stochastic Methods, 3ed. (Springer, Berlin, 2004).

${ }^{12}$ J. D. Gunton, A. Shiryayev, and D. L. Pagan, Protein Condensation: Kinetic Pathways to Crystallization and Disease (Cambridge University Press, Cambridge, 2007).

${ }^{13}$ P. R. ten Wolde and D. Frenkel, Science 77, 1975 (1997).

${ }^{14}$ J. F. Lutsko, J. Chem. Phys. 134, 164501 (2011).

${ }^{15}$ J. A. Barker and D. Henderson, J. Chem. Phys. 47, 4714 (1967).

${ }^{16}$ Y. Saito, Statistical Physics of Crystal Growth (World Scientific, Singapore, 1998).

${ }^{17}$ M. J. Uline and D. S. Corti, Phys. Rev. Lett. 99, 076102 (2007).

${ }^{18}$ S. Ghosh and S. K. Ghosh, J. Chem. Phys. 134, 024502 (2011).

${ }^{19}$ A. J. Archer and R. Evans, Molecular Physics 109, 2711 (2011). 
${ }^{20}$ J. F. Lutsko, Europhys. Lett. 83, 46007 (2008).

${ }^{21}$ J. F. Lutsko, J. Chem. Phys. 129, 244501 (2008).

${ }^{22}$ T. Philippe and D. Blavette, J. Chem. Phys. 135, 134508 (2011).

${ }^{23}$ C. Qiu and T. Qian, J. Chem. Phys. 131, 124708 (2009).

${ }^{24}$ J. F. Lutsko, Adv. Chem. Phys. 151, 137 (2012).

${ }^{25}$ X. Cheng, L. Lin, W. E, P. Zhang, and A.-C. Shi, Phys. Rev. Lett. 104, 148301 (Apr 2010).

${ }^{26}$ M. Robbins, A. Archer, and U. Thiele, J. Phys.: Condens. Matter 23, 415102 (2011).

${ }^{27}$ J. Zinn-Justin, Quantum Field Theory and Critical Phenomena (Oxford Univ. Press, Oxford, 2003).

${ }^{28}$ W. H. Press, S. A. Teukolsky, W. T. Vetterling, and B. P. Flannery, Numerical Recipes in C (Claredon Press, Oxford, 1993).

${ }^{29}$ D. Wales, Energy Landscapes (Cambridge University Press, Cambridge, 2003). 